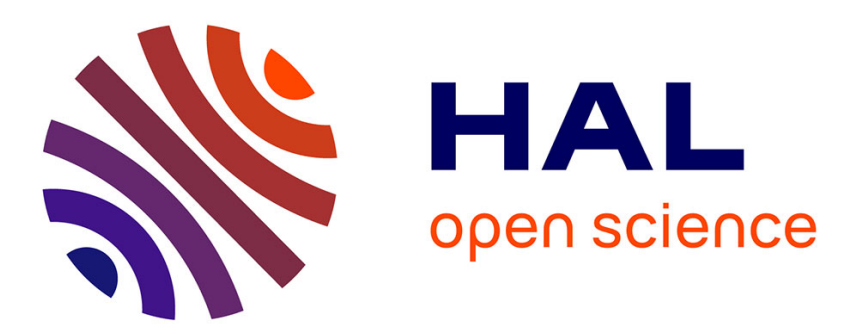

\title{
Numerical and experimental determination of the thermal conductivity of pristine and substituted Fe2VAl
}

Martin Noutack, Abou Diack-Rasselio, Eric Alleno, Philippe Jund

\section{To cite this version:}

Martin Noutack, Abou Diack-Rasselio, Eric Alleno, Philippe Jund. Numerical and experimental determination of the thermal conductivity of pristine and substituted Fe2VAl. Journal of Alloys and Compounds, 2021, 883, pp.160828. 10.1016/j.jallcom.2021.160828 . hal-03268331

\section{HAL Id: hal-03268331 \\ https://hal.science/hal-03268331}

Submitted on 17 Nov 2021

HAL is a multi-disciplinary open access archive for the deposit and dissemination of scientific research documents, whether they are published or not. The documents may come from teaching and research institutions in France or abroad, or from public or private research centers.
L'archive ouverte pluridisciplinaire HAL, est destinée au dépôt et à la diffusion de documents scientifiques de niveau recherche, publiés ou non, émanant des établissements d'enseignement et de recherche français ou étrangers, des laboratoires publics ou privés. 


\title{
Numerical and experimental determination of the thermal conductivity of pristine and substituted $\mathrm{Fe}_{2} \mathrm{VAl}$
}

\author{
Martin S. Talla Noutack ${ }^{1}$, Abou Diack-Rasselio ${ }^{2}$, Eric Alleno ${ }^{2}$ and Philippe Jund ${ }^{1 *}$ \\ ${ }^{1}$ ICGM, Univ. Montpellier, CNRS, ENSCM, Montpellier, France \\ ${ }^{2}$ Univ. Paris Est Creteil, CNRS, ICMPE, UMR 7182, 2-8, rue H. Dunant, F-94320 THIAIS, France
}

March 2, 2021

\begin{abstract}
In this article, we investigate the lattice thermal conductivity in the thermoelectric $\mathrm{Fe}_{2} \mathrm{VAl}$ Heusler alloy by implementing a Green's function approach to describe phonondefect scattering mechanisms in the pristine and substituted compounds. Former calculations for pristine $\mathrm{Fe}_{2} \mathrm{VAl}$ largely overestimated the lattice thermal conductivity compared to the experimental value $\left(28 \mathrm{Wm}^{-1} \mathrm{~K}^{-1}\right)$. By considering a realistic concentration $(\sim 5$ $\%$ ) of combined $\mathrm{Al}_{V}$ and $\mathrm{V}_{A l}$ antisite defects as an extra phonon scattering mechanism, calculated and experimental values could be reconciled. The same theoretical approach was applied to $\mathrm{Ta}$ - and $\mathrm{Ta}+\mathrm{Sn}$-substituted $\mathrm{Fe}_{2} \mathrm{VAl}$. In agreement with experiments it shows that the dual Ta + Sn-substitution is more efficient in decreasing the lattice thermal conductivity of $\mathrm{Fe}_{2} \mathrm{VAl}$ than the single Ta-substitution. A strong decrease of $70 \%$ is theoretically obtained in $\mathrm{Fe}_{2} \mathrm{~V}_{0.96} \mathrm{Ta}_{0.04} \mathrm{Al}_{0.96} \mathrm{Sn}_{0.04}$ which could thus lead to better global thermoelectric performances .
\end{abstract}

\section{Introduction}

Thermoelectric devices attract much of interest for their potential application as electrical generators. In particular, they could be used in factories for recycling wasted heat [1] or in automobiles for increasing the fuel efficiency [2]. The thermoelectric efficiency of a material is evaluated by a dimensionless quantity called the figure of merit $Z T=\frac{\sigma S^{2}}{\kappa} T$, where $T$ is the temperature, $S$ the Seebeck coefficient, $\sigma$ the electrical conductivity and $\kappa$ the thermal conductivity consisting in both lattice $\left(\kappa_{\text {lat }}\right)$ and electronic $\left(\kappa_{e}\right)$ contributions. Bismuth telluride $\left(\mathrm{Bi}_{2} \mathrm{Te}_{3}\right)$ is the reference material currently used in commercialized thermoelectric devices and it displays $\mathrm{ZT}=1$ at $300 \mathrm{~K}$. Over the last decade, full Heusler compounds, more specifically $\mathrm{Fe}_{2} \mathrm{VAl}$ has been considered as a potential contender to $\mathrm{Bi}_{2} \mathrm{Te}_{3}$. Well-doped $\mathrm{Fe}_{2} \mathrm{VAl}$ displays values of power factor $P F=\sigma S^{2}$ in the range $5-10 \mathrm{mWm}^{-1} \mathrm{~K}^{-2}$ at $300 \mathrm{~K}[3,4,5]$, well beyond the value found in $\mathrm{Bi}_{2} \mathrm{Te}_{3}\left(5 \mathrm{mWm}^{-1} \mathrm{~K}^{-2}\right)$. However, the lattice thermal conductivity of $\mathrm{Fe}_{2} \mathrm{VAl}$ is rather large, typically $\kappa_{\text {lat }}=28 \mathrm{Wm}^{-1} \mathrm{~K}^{-1}$ at $300 \mathrm{~K}$ in the pristine compound, which is a value more than an order of magnitude larger than in $\mathrm{Bi}_{2} \mathrm{Te}_{3}\left(\kappa_{\text {lat }}=1.3 \mathrm{Wm}^{-1} \mathrm{~K}^{-1}\right)$ [6]. Its figure of merit is thus an order of magnitude smaller than in $\mathrm{Bi}_{2} \mathrm{Te}_{3}$ and lowering the lattice thermal conductivity is required to improve the thermoelectric efficiency of $\mathrm{Fe}_{2} \mathrm{VAl}$. This can be achieved by introducing "obstacles" to the phonon-heat transport by mainly two different ways: i) introducing nanoprecipitates or grain boundaries by nanostructuring; ii) substituting

*philippe.jund@umontpellier.fr 
one or several constituting element(s) by another one with a different mass or "alloying". Concerning this last way, several experimental studies $[4,7,8,9]$ reported in the literature show that substituting by heavier chemical elements can lead to an important decrease of the lattice thermal conductivity. Several experimental studies report $\kappa_{\text {lat }}$ in $\mathrm{Fe}_{2} \mathrm{VAl}$ ranging between $23-28 \mathrm{Wm}^{-1} \mathrm{~K}^{-1}$ at $300 \mathrm{~K}[9,10]$ whereas theoretical approaches combining a DFT method with either the resolution of the Boltzmann transport equation [11] or with an approximated Debye-Callaway model [12] calculated $\kappa_{\text {lat }}=58 \mathrm{Wm}^{-1} \mathrm{~K}^{-1}$, thus largely overestimating the experimental values. In ref. [12], another value was calculated for the lattice thermal conductivity $\left(\kappa_{\text {lat }}=21 \mathrm{Wm}^{-1} \mathrm{~K}^{-1}\right)$ but at the expense of using a non DFT-relaxed unit-cell volume. Given that several ab initio studies on semiconductors $[13,14,15]$ show a relatively good agreement with experimental results, this discrepancy between theory and experiment in $\mathrm{Fe}_{2} \mathrm{VAl}$ is not well understood and needs to be addressed as a question. Moreover, only one $\kappa_{\text {lat }}$ calculation in substituted $\mathrm{Fe}_{2} \mathrm{VAl}\left(\mathrm{Fe}_{1.75} \mathrm{Ni}_{0.25} \mathrm{VAl}\right)$ is reported in the literature [12] giving the same overestimation as in pristine $\mathrm{Fe}_{2} \mathrm{VAl}$, when compared to experiment. This other question also needs to be addressed, not only from a theoretical point of view but also from the point of view of the experiments that need a guide to undertake the "alloying" research way, e.g. to introduce mass fluctuations in the lattice by substitution. By supplying predictive evaluations, theory can help select the most suitable combination of substituted elements to efficiently decrease $\kappa_{\text {lat }}$.

To answer the first question, the present work considers the role of $\mathrm{Al} / \mathrm{V}$ inversions in calculating the lattice thermal conductivity of $\mathrm{Fe}_{2} \mathrm{VAl}$. We have shown in a previous theoretical study [16] that $\mathrm{Al} / \mathrm{V}$ inversions (a combination of $A l_{V}$ and $V_{A l}$ antisite defects) are the most probable defects to form at high temperature. Experimentally, even strictly stoichiometric $\mathrm{Fe}_{2} \mathrm{VAl}$ samples most likely contain a sizable concentration of $A l_{V}$ and $V_{A l}$ antisite defects, which depends on their thermal history (for instance their annealing temperature when $\mathrm{T}>$ $800{ }^{\circ} \mathrm{C}$ ) since at $\mathrm{T}=1080{ }^{\circ} \mathrm{C}, \mathrm{Fe}_{2} \mathrm{VAl}$ undergoes an order-disorder transition (second order) where $50 \%$ of $\mathrm{V}$ and $50 \%$ of $\mathrm{Al}$ atoms mix on their respective crystallographic sites [17]. As a consequence, $\mathrm{Al} / \mathrm{V}$ inversions could play an important role in lowering the lattice thermal conductivity by introducing an additional phonon scattering mechanism such as $\mathrm{Al} / \mathrm{V}$ mass fluctuations.

To address the question of extrinsic substitution, the lattice thermal conductivity is calculated in Ta- and $\mathrm{Ta}+\mathrm{Sn}$-substituted $\mathrm{Fe}_{2} \mathrm{VAl}$. It is indeed already known experimentally that vanadium substitution by tantalum efficiently decreases $\kappa_{\text {lat }}[4,37]$ but no theoretical confirmation has yet been performed. Moreover, we showed in a preliminary study (not yet published) that tantalum can improve the electronic part of the figure of merit in $\mathrm{Fe}_{2} \mathrm{VAl}$. Tantalum is thus an obvious candidate for theoretically studying its alloying effect on $\kappa_{\text {lat }}$. The effect of tin is not better known: our recent study (unpublished), only experimental, shows that $\kappa_{\text {lat }}$ decreases upon its substitution on the aluminum site, as expected from the large mass difference of these two atoms. It is thus tempting to substitute both atoms on the $\mathrm{V}$ - and $\mathrm{Al}$ - site in $\mathrm{Fe}_{2} \mathrm{VAl}$ to combine their effects on $\kappa_{\text {lat }}$. Nonetheless, given the change in the interatomic forces constants (IFCs) most likely induced by substitution, simple intuition cannot predict if the dual $\mathrm{Ta}+\mathrm{Sn}$ substitution will decrease more $\kappa_{\text {lat }}$ than the single Ta substitution. We thus rigorously compared these two situations by carrying out calculations on $\mathrm{Fe}_{2} \mathrm{~V}_{1-2 x} \mathrm{Ta}_{2 x} \mathrm{Al}$ and $\mathrm{Fe}_{2} \mathrm{~V}_{1-x} \mathrm{Ta}_{x} \mathrm{Al}_{1-x} \mathrm{Sn}_{x}$, every pair of compositions displaying the same total concentration of substituents. This was followed by measurements of $\kappa_{\text {lat }}$ in $\mathrm{Fe}_{2} \mathrm{~V}_{0.94} \mathrm{Ta}_{0.06} \mathrm{Al}$ and $\mathrm{Fe}_{2} \mathrm{~V}_{0.97} \mathrm{Ta}_{0.03} \mathrm{Al}_{0.97} \mathrm{Sn}_{0.03}$ to check the theoretical predictions.

The calculation of the phonon-defect scattering rate is now possible thanks to the recently developed $a b$ initio Green's function approach $[18,19]$ that we will be using in the present study. Our computational approach and experimental methods are described in Sec. 2. After presenting the role of the $\mathrm{Al} / \mathrm{V}$ inversions in lowering $\kappa_{\text {lat }}$, experimental and calculated data for stoichiometric $\mathrm{Fe}_{2} \mathrm{VAl}$ are compared in Sec. 3. The effect of substituting Ta and Sn on $\kappa_{\text {lat }}$ is examined in Sec. 4. Finally, in Sec. 5 we draw the major conclusions of this work. 


\section{Computational details and experimental methods}

\subsection{Heat conduction in defective compounds}

Two approaches can be used to obtain the lattice thermal conductivity in defective compounds. The first one consists in assuming that the presence of defects modifies all the lattice phonons and thus the harmonic and anharmonic interatomic forces constants. In practice, defects are created in a supercell before performing finite displacements calculations which permit then to compute the IFCs. However, given the large number of irreducible triplets involved in the calculation of the phonon scattering rates (through three-phonon processes), this approach needs a very long calculation time (several months on a supercomputer depending on the size of the supercell). The second approach is based on the Green's function, which consists in considering that the phonons of the perfect cell only locally interact with the defect. The defect is seen as a local perturbation that modifies the interatomic force constants only in a small region around the defect. This approach has already been used in the literature to study the thermal conductivity in defective compounds $[11,18,19,20,21]$. The calculation of the phonon-defect scattering rate $\left(\tau^{d e f}\right)$ is implemented in the code developed within the ALMA project [22]. The $1 / \tau^{\text {def }}$ expression is based on an atomistic description of each defect considering the $a b$ initio Green's function approach [18]. Using the Lippmann-Schwinger equation, $1 / \tau^{d e f}$ can be expressed as follow:

$$
\frac{1}{\tau_{q s}^{d e f}}=x_{d e f} \frac{\Omega}{V_{d e f}} \frac{\pi}{\omega_{q s}} \sum_{q^{\prime} s^{\prime}}\left|\left\langle q^{\prime} s^{\prime}\left|\left(I-\hat{V} g^{+}\right)^{-1} \hat{V}\right| q s\right\rangle\right|^{2} \delta\left(\omega_{q^{\prime} s^{\prime}}^{2}-\omega_{q s}^{2}\right)
$$

where $|q s\rangle$ and $\left|q^{\prime} s^{\prime}\right\rangle$ are the incident and outgoing phonons (with wave vector $q$ and belonging to band $s$ ), $x_{\text {def }}$ is the concentration of defects i.e. the number of defects divided by the number of available sites. Defects are supposed to be uncorrelated for a given defect type. $V_{d e f}$ and $\Omega$ are the volume of each defect and the volume in which $|q s\rangle$ is normalized, respectively. $\omega$ is the angular frequency of the phonon. $I$ is the identity matrix, $\hat{V}$ the perturbation matrix and $g^{+}$ the Green's function of the unperturbed system (perfect compound).

Generally, $\hat{V}=\hat{V}_{M}+\hat{V}_{K}$ where $\hat{V}_{M}$ and $\hat{V}_{K}$ are the perturbation related to the mass term and the harmonic IFC term respectively. These two terms can be expressed as follow:

$\hat{V}_{M}=-\frac{M_{i}^{\prime}-M_{i}}{M_{i}} \omega^{2}$ and $\hat{V}_{K}=\frac{K_{i \alpha, j \beta}^{\prime}-K_{i \alpha, j \beta}}{\left(M_{i} M_{j}\right)^{1 / 2}}$, where $M_{i}$ and $M_{i}^{\prime}$ refer to corresponding atomic masses for the unperturbed and perturbed system respectively, $K$ and $K^{\prime}$ refer to the corresponding harmonic IFCs for the unperturbed and perturbed system respectively. In this study, $i$ correspond to all atoms lying in a cluster of radius $\sim 5.5 \AA$ around the defect and surrounded (first and second nearest atoms) by atoms $j . \alpha$ and $\beta$ are the space directions in which atoms are displaced.

\subsection{Lattice thermal conductivity}

The lattice thermal conductivity $\kappa_{\text {lat }}$ is calculated through the Boltzmann transport equation within the relaxation time approximation. For an isotropic system, $\kappa_{\text {lat }}$ is given by:

$$
\kappa_{\text {lat }}=\frac{1}{3 V N} \sum_{q, s} C_{q s} \nu_{q s}^{2} \tau_{q s}
$$

where $C_{q s}, \nu_{q s}$ represents the specific heat and phonon group velocity of the unperturbed system respectively, and $\tau_{q s}$ is the phonon relaxation time for each phonon mode qs. $V$ is the volume of the primitive cell and $N$ is the number of q points in the irreducible Brillouin zone.

In an unperturbed system the main source of the phonon scattering is the anharmonicity which is usually dominated by the three-phonon processes with the scattering rate denoted $\tau_{q s}^{p h-p h}$. 
Another source of scattering comes from the isotopic contribution, the corresponding scattering rate being termed $\tau_{q s}^{\text {iso }}$ (see Ref. [23, 24]).

The boundary effect in the lattice thermal conductivity has also been considered using a standard formula based on the Casimir approximation:

$$
\frac{1}{\tau_{q s}^{\text {grain }}}=\frac{\nu_{q s}}{L_{\text {grain }}}
$$

where $\nu_{q s}$ is the group velocity and $L_{\text {grain }}$ the grain size.

In a defective compound, an additional scattering rate $\left(\tau_{q s}^{\text {def }}\right)$ due to the phonon-defect scattering (as described in the previous section (Sec. 2.1)) has to be considered. Finally the total scattering rate $\tau_{q s}$ is obtained using the Matthiessen rule:

$$
\frac{1}{\tau_{q s}}=\frac{1}{\tau_{q s}^{p h-p h}}+\frac{1}{\tau_{q s}^{\text {iso }}}+\frac{1}{\tau_{q s}^{\text {grain }}}+\frac{1}{\tau_{q s}^{\text {def }}}
$$

The calculation of the lattice thermal conductivity in this work is performed using the AlmaBTE code [22]. We have compared the lattice thermal conductivity, including only threephonon and isotope scattering, calculated from the AlmaBTE and PHONO3PY codes [25] in $\mathrm{Fe}_{2}$ VAl. Fig. 1 shows that a very good agreement between both codes was obtained.

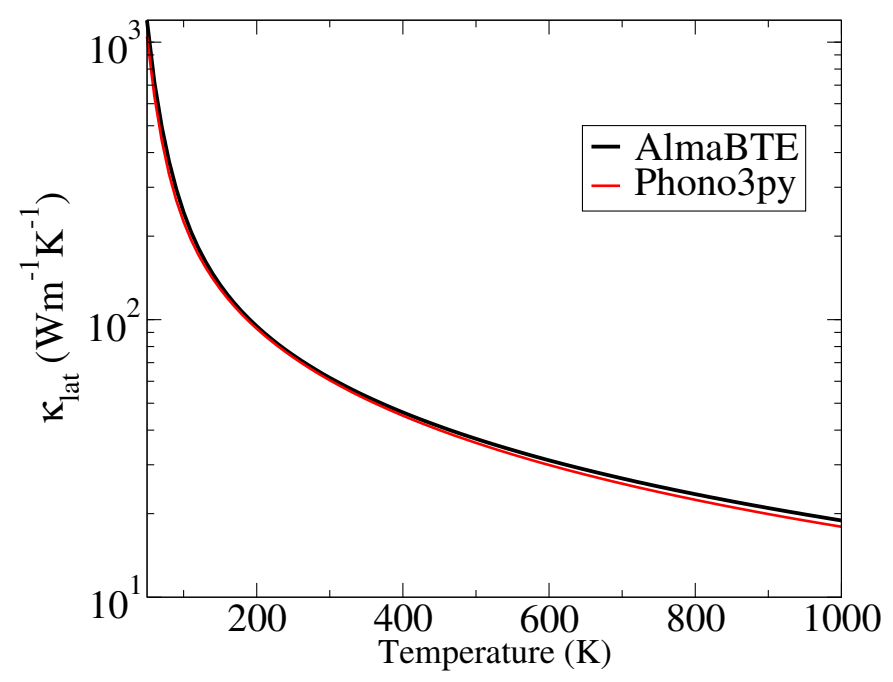

Figure 1: AlmaBTE and Phono3py lattice thermal conductivities. A q-point mesh of $11 \times 11 \times 11$ is used in both cases. Only phonon-phonon and isotope scattering mechanisms are considered.

\subsection{First-principles calculation of the force constants}

Hellmann-Feynmann forces have been obtained within the density functional theory (DFT) framework as implemented in the VASP package [26]. The exchange-correlation functional has been described using the generalized gradient approximation (GGA) as parametrized by Perdew, Burke and Enzerhof [27].

All the results have been obtained using a plane-wave cutoff energy of $500 \mathrm{eV}$. According to our convergence tests, this parameter leads to a precision lower than 1 meV per atom on physical energies (total energy differences). A 128-atom $2 \times 2 \times 2$ supercell of the conventional fcc full-Heusler cell of $\mathrm{Fe}_{2} \mathrm{VAl}$ ( $\mathrm{L}_{1}$ structure type) has been considered in this study.

The calculations have been done using a $2 \times 2 \times 2 \mathrm{k}$-point mesh to sample the Brillouin zone associated to the supercell, generated by the Monkhorst-Pack [28] method, which is sufficient for an energy convergence lower than $0.1 \mathrm{meV}$ per atom in the supercell. The third-order interatomic force constants have been obtained using the finite displacements method on the 
128 atoms of the supercell. Atoms are displaced by pairs in the three directions of space taking symmetry into account and considering a cutoff distance corresponding to the fifth nearest-neighbors distance. During the calculation of the forces acting on the atoms (after performing the finite displacements), the volume and the positions are kept fixed and the energy convergence criterion is set to $10^{-8} \mathrm{eV}$.

The second-order force constants have been extracted using the PHONOPY package [29] from the Hessian matrix calculated by the density functional perturbation theory (DFPT) method as implemented in the VASP package. Note that before performing DFPT, the supercells are first fully relaxed (volume and atomic positions) until the energy is converged to $10^{-7}$ eV per atom.

In order to evaluate the influence of the q mesh on the lattice thermal conductivity of pristine L2 $\mathrm{Fe}_{2} \mathrm{VAl}$, we performed four preliminary calculations with $\mathrm{q}$ mesh sizes of: $11 \times 11 \times 11$, $15 \times 15 \times 15,19 \times 19 \times 19$, and $25 \times 25 \times 25$. The results indicate that a q-point mesh of $11 \times 11 \times 11$ is sufficient to achieve the convergence of the lattice thermal conductivity $\kappa_{\text {lat }}$ for $\mathrm{T}>50 \mathrm{~K}$.

\subsection{Experimental}

$\mathrm{Fe}_{2} \mathrm{VAl}$ and the Ta- and ( $\mathrm{Ta}+\mathrm{Sn}$ )-substituted samples $\mathrm{Fe}_{2} \mathrm{~V}_{0.94} \mathrm{Ta}_{0.06} \mathrm{Al}$ and $\mathrm{Fe}_{2} \mathrm{~V}_{0.97} \mathrm{Ta}_{0.03} \mathrm{Al}_{0.97} \mathrm{Sn}_{0.03}$ were prepared using appropriate amounts of high purity iron (99.97\%), vanadium (99.99 \%), aluminum $(99.98 \%)$, tantalum $(99.96 \%)$ and tin $(99.9 \%)$ in an arc-melting furnace. The samples were repeatedly melted and flipped over to ensure homogeneity. They were subsequently cut using a wheel saw (Buehler) and finely polished to obtain a disk of constant thickness $( \pm 0.02 \mathrm{~mm})$. To anneal and at the same time release microstrains caused by cutting and polishing, the samples were sealed in an evacuated quartz tube, heated for $72 \mathrm{~h}$ at $1173 \mathrm{~K}$ and furnace cooled. An average grain size of $L_{\text {grain }}=280 \mu \mathrm{m}$ was derived from observations of the microstructure performed with a scanning electron microscope (CAMECA SX100). Thermal diffusivity (a) was measured under an argon atmosphere from $300 \mathrm{~K}$ to $600 \mathrm{~K}$ by the laser flash method using a Netzsch LFA 457 equipment. The thermal conductivity $(\kappa)$ was derived using the relationship $\kappa=a \times C_{p} \times d$, with $C_{p}$ the specific heat capacity and $d$ the measured density (Archimède's method). The specific heat was derived using Dulong and Petit's law. The electrical conductivity $(\sigma)$ was measured in an argon atmosphere from $300 \mathrm{~K}$ to $600 \mathrm{~K}$ using a home-made apparatus, described in detail by Rouleau et al in ref. [30]. The electronic contribution of the thermal conductivity was derived using the Wiedemann-Franz law $\kappa_{e}=L \times T \times \sigma$, with $L=1.8 \times 10^{-8} V^{2} K^{-2}$, the Lorentz number and $T$ the temperature. $\kappa_{\text {lat }}$ was taken as the difference $\kappa-\kappa_{e}$, neglecting a possible bipolar contribution. Room temperature Hall effect measurements were performed in a Physical Properties Measurement System (PPMS, Quantum Design) in AC mode by varying the magnetic field between $-3 \mathrm{~T}$ and $+3 \mathrm{~T}$. The measured apparent hole concentration was defined by $p_{m}=1 /\left(e \times R_{H m}\right)$, with $e$ the elementary charge and $R_{H m}$ the measured Hall coefficient.

\section{Lattice thermal conductivity of $\mathrm{Fe}_{2} \mathrm{VAl}$ and role of $\mathrm{Al} / \mathrm{V}$ inversions}

A first calculation of $\kappa_{\text {lat }}$ was performed in perfectly crystallized $\mathrm{Fe}_{2} \mathrm{VAl}\left(\mathrm{L} 2_{1}\right.$ structure). It is compared in Fig. 2 with experimental results, measured in the present study as well as retrieved from the literature.

We mainly focused on the temperature range between $300 \mathrm{~K}$ and $600 \mathrm{~K}$, which is the interesting range for thermoelectric applications. One can notice that the experimental lattice thermal conductivity, especially at $300 \mathrm{~K}$, varies between $23-28 \mathrm{Wm}^{-1} \mathrm{~K}^{-1}$ depending on the samples. There is nonetheless a convergence of several experimental values towards $\kappa_{\text {lat }}=28 \mathrm{Wm}^{-1} \mathrm{~K}^{-1}$ at $300 \mathrm{~K}$. Our calculations, taking only phonon-phonon, isotope and boundary scattering into 


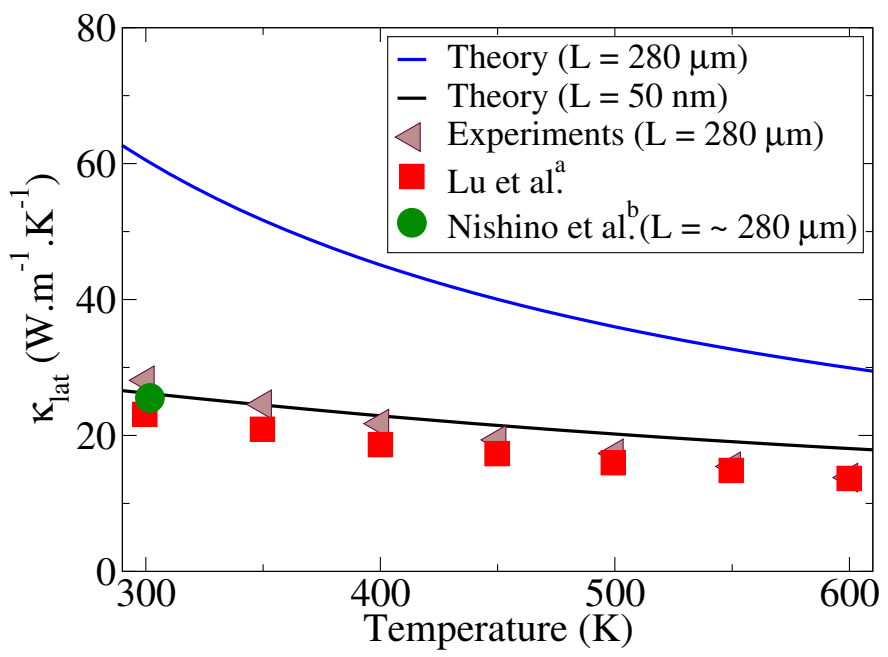

Figure 2: Thermal conductivity of perfect $\mathrm{Fe}_{2} \mathrm{VAl}$, where three-phonon, isotope and boundary scattering mechanisms are considered in Eq. 4 calculated using the single-mode relaxation-time approximation as implemented in almaBTE [22]. For comparison, the experimental values from the literature and from the present work are also displayed.

${ }^{a}$ Ref.[10], ${ }^{b}$ Ref.[8].

account lead to $\kappa_{\text {lat }}$ equals to $60 \mathrm{Wm}^{-1} \mathrm{~K}^{-1}$ at $300 \mathrm{~K}$ (for a grain size of $L_{\text {grain }}=280 \mu \mathrm{m}$ ), thus largely overestimating the lattice thermal conductivity. This result is nevertheless in agreement with previously calculated values by Bandaru et al. [11], considering the same scattering mechanisms and a larger P444 supercell (256 atoms). Moreover, one can notice in Fig. 2 that in order to reproduce the experimental data, the grain size has to be drastically reduced to 50 $\mathrm{nm}$. However, this grain size is too small compared to the reported and measured experimental sizes and thus, cannot justify the disagreement between experiments and theory. This discrepancy is surprising since it has been clearly shown in the literature that by only considering phonon-phonon and isotope scattering, reliable values of $\kappa_{\text {lat }}$ in semiconductors $[13,14,15]$ can be obtained. Note that this poor agreement between experiment and calculations has already been reported in the case of $\mathrm{FeSi}$ [33] and $\mathrm{FeSb}_{2}$ [34]. Such a disagreement indicates that further scattering mechanisms, in addition to phonon-phonon, isotope and boundary scattering have to be taken into account in order to lower $\kappa_{\text {lat }}$. The thermal history of the samples considered in experiments may play an important role in assessing their thermal conductivity. Indeed, depending on its annealing temperature and quenching conditions, a sample may contain a certain concentration of point defects. Consequently, these defects can lower the lattice thermal conductivity as a result of phonon-defect scattering. We suspect the scattering by $\mathrm{Al} / \mathrm{V}$ inversions to play an important role in lowering $\kappa_{\text {lat }}$ in $\mathrm{Fe}_{2} \mathrm{VAl}$. $\mathrm{Al} / \mathrm{V}$ inversions are defects leaving the composition unchanged and resulting from the interchange of nearest neighbor $\mathrm{Al}$ and $\mathrm{V}$ sites. They can also be seen as two combined $\mathrm{Al}_{V}$ and $\mathrm{V}_{A l}$ antisite defects. Since their formation energy is equal to $0.34 \mathrm{eV}$ [16], they are the most likely thermally activated defects as shown in experiments [17] and simulations [16] and thus can be formed during annealing at high temperature $\mathrm{T}>800{ }^{\circ} \mathrm{C}$. Moreover, in our previous study [16], we showed that by taking $\mathrm{Al} / \mathrm{V}$ inversions and temperature effects into account, the disagreement between the experimental and calculated band gap of $\mathrm{Fe}_{2} \mathrm{VAl}$ at high temperature $(400-800 \mathrm{~K})$ can be reduced, indicating that inversions are probably present in experimental samples. For the calculation of the perturbation matrix $\hat{V}$ (see Eq. 1), we have considered changes in the harmonics IFCs for first- and second-nearest-neighbour interactions of all the atoms lying in a cluster of radius $\sim 5.5 \AA$ around the defect. The defect concentration introduced in our 128-atoms supercell is $x_{\text {def }} \sim 3 \%$, corresponding to interchanging $1 \mathrm{Al}$ atom and $1 \mathrm{~V}$ atom on the 64 available sites. In Figure 3, we investigate the influence of the concentration of inversions on the lowering of $\kappa_{\text {lat }}$ due to additional defect scattering. 


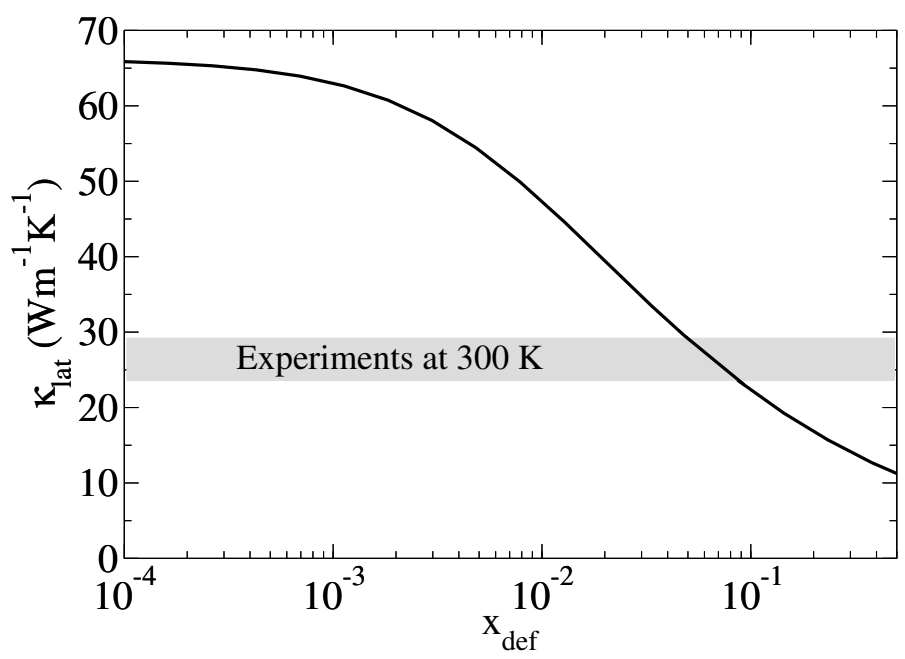

Figure 3: Lattice thermal conductivity of $\mathrm{Fe}_{2} \mathrm{VAl}$ at $300 \mathrm{~K}$ as a function of $x_{\text {def }}$, the concentration of $\mathrm{Al} / \mathrm{V}$ inversions as defined in Eq. 1 (a grain size of $280 \mu \mathrm{m}$ has been considered.)

Firstly, $\kappa_{\text {lat }}$ displays a complex dependence on $x_{\text {def }}$ and secondly, a significant lowering of the lattice thermal conductivity is observed, starting from a defect concentration around $x_{d e f}$ $\approx 0.1 \%$ corresponding to $C_{d e f} \approx 2.4 \times 10^{19} \mathrm{~cm}^{-3}$. Furthermore, one can see that for a defect concentration around $x_{\text {def }} \approx 5 \%$ corresponding to $C_{\text {def }} \approx 1.1 \times 10^{21} \mathrm{~cm}^{-3}$, the lattice thermal conductivity is predicted to be $29 \mathrm{Wm}^{-1} \mathrm{~K}^{-1}$, which is in good agreement with the experimental values. This agreement is confirmed not only at $300 \mathrm{~K}$ but also at higher temperature in Figure 4, where the lattice thermal conductivity for various defect concentrations is compared with the experimental values as a function of temperature: the $x_{d e f}=5 \%$ calculated curve matches the present experiments.

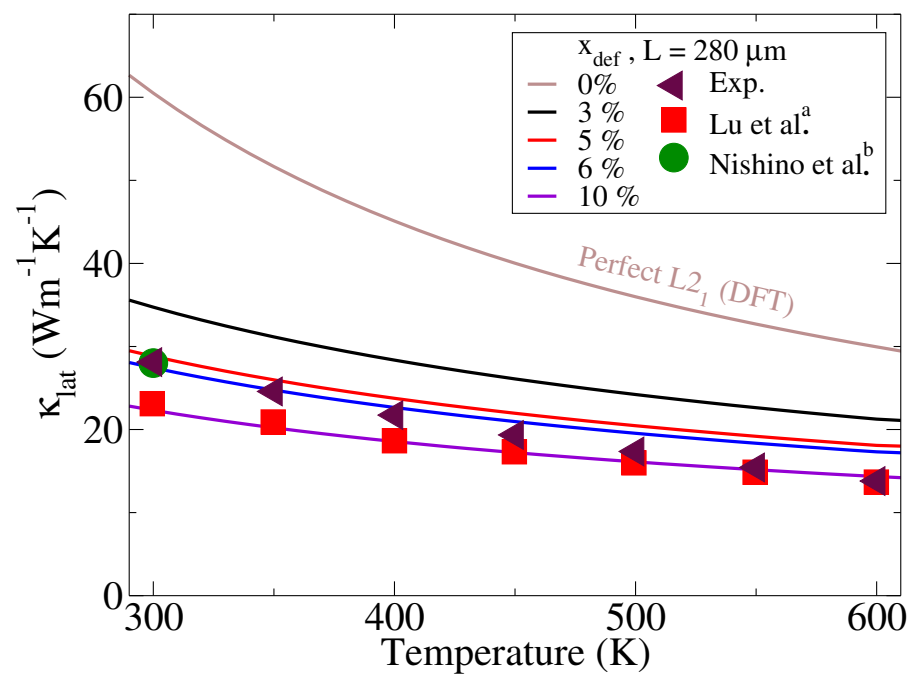

Figure 4: Lattice thermal conductivity of $\mathrm{Fe}_{2} \mathrm{VAl}$ versus temperature for different $\mathrm{Al} / \mathrm{V}$ inversion concentrations. The experimental grain size is approximately equal to $280 \mu \mathrm{m}$ in the present experiment.

${ }^{a} \operatorname{Ref} .[10],{ }^{b} \operatorname{Ref} .[8]$.

An estimation of the experimental concentration of defects can be made by considering Hall effect data. In the stoichiometric sample of Fig. 2, the measured apparent hole concentration at $300 \mathrm{~K}$ is $p_{m}=7 \times 10^{20} \mathrm{~cm}^{-3}$. We have previously shown that an $V_{A l}$ is a 1 electron donor whereas a $A l_{V}$ is a 1 electron acceptor [31]. Assuming that only $\mathrm{Al} / \mathrm{V}$ inversions are present in this stoichiometric sample and that both defects are fully ionized at $300 \mathrm{~K}$, then $n=\left[V_{A l}\right]=p$ $=\left[A l_{V}\right]$ with $n$ and $p$ the actual electron and hole concentrations respectively. In a situation where two kinds of carriers coexist, the measured apparent Hall coefficient is given by the 
relation [32]:

$$
R_{H m}=\frac{1}{e} \frac{p \mu_{p}^{2}-n \mu_{n}^{2}}{\left(p \mu_{p}+n \mu_{n}\right)^{2}}
$$

with $e$ the elementary charge, $\mu_{p}$ and $\mu_{n}$ are the hole and electron mobility respectively. Setting $\mathrm{r}=\mu_{p} / \mu_{n}$ and using $p=n$, and $\mathrm{p}=\left[A l_{V}\right]$ in equation 5 leads to:

$$
\left[A l_{V}\right]=p_{m} \frac{r-1}{r+1}
$$

Our unpublished mobility measurements in hole- and electron-doped $\mathrm{Fe}_{2} \mathrm{VAl}$ have yielded $\mathrm{r}=3.6$. The estimated concentration of $\mathrm{Al} / \mathrm{V}$ inversions in the $\mathrm{Fe}_{2} \mathrm{VAl}$ sample of Fig. 3 is thus $\left[A l_{V}\right]=4 \times 10^{20} \mathrm{~cm}^{-3}$ or $x_{d e f-\exp }=1.9 \%$. The order of magnitude of this experimental estimation of $x_{\text {def-exp }}$ agrees with $x_{d e f-c a l}=5 \%$ deduced from the calculations of $\kappa_{\text {lat }}$ (see Fig. 4). The difference between the two values may arise from uncertainties in the calculated value and from the fact that one assumption made to deduce the experimental $x_{\text {def-exp }}$ from the Hall data may not be entirely fulfilled: the full ionization of the $\mathrm{Al} / \mathrm{V}$ defects may not be completed at $300 \mathrm{~K}$, explaining why $x_{d e f-e x p}<x_{d e f-c a l}$. Nonetheless, our results undeniably show that point defect scattering of phonons by $\mathrm{Al} / \mathrm{V}$ inversions play a central role in explaining the lattice thermal conductivity in pristine $\mathrm{Fe}_{2} \mathrm{VAl}$.

\section{Lattice thermal conductivity in Ta- and $\mathrm{Ta}+\mathrm{Sn}-$ substituted $\mathrm{Fe}_{2} \mathrm{VAl}$}

After evaluating the role of $\mathrm{Al} / \mathrm{V}$ inversions on the lattice thermal conductivity of $\mathrm{Fe}_{2} \mathrm{VAl}$, we now tackle the effect of substitutions by other chemical elements. Table 1 compiles the formation energies and most probable charge of $\mathrm{X}(\mathrm{X}=\mathrm{Ta}, \mathrm{Sn})$ in $\mathrm{Fe}_{2} \mathrm{~V}_{0.97} \mathrm{X}_{0.03} \mathrm{Al}$ and $\mathrm{Fe}_{2} \mathrm{VAl}_{0.97} \mathrm{X}_{0.03}$.

\begin{tabular}{lcc}
\hline \hline Compounds & charge (q) & Formation energy (eV) \\
\hline $\mathrm{Fe}_{2} \mathrm{VAl}_{0.97} \mathrm{Ta}_{0.03}$ & +2 & 1.22 \\
$\mathrm{Fe}_{2} \mathrm{~V}_{0.97} \mathrm{Ta}_{0.03} \mathrm{Al}$ & 0 & 0.10 \\
$\mathrm{Fe}_{2} \mathrm{~V}_{0.97} \mathrm{Sn}_{0.03} \mathrm{Al}$ & -1 & 1.96 \\
$\mathrm{Fe}_{2} \mathrm{VAl}_{0.97} \mathrm{Sn}_{0.03}$ & +1 & 1.77 \\
\hline \hline
\end{tabular}

Table 1: Most probable charge and formation energies of Ta and Sn substitutional defects calculated using the expression detailed in [31] taking into account the corrections related to charged defects as detailed in [36].

These compositions correspond to the substitution of one $\mathrm{V}$ atom or one $\mathrm{Al}$ atom in a $2 \times 2 \times 2$ conventional supercell (128 atoms) by one $\mathrm{X}$ atom, which is considered as an extrinsic defect. In the case of $\mathrm{Ta}+\mathrm{Sn}$-substituted $\mathrm{Fe}_{2} \mathrm{VAl}\left(\mathrm{Fe}_{2} \mathrm{~V}_{0.97} \mathrm{Ta}_{0.03} \mathrm{Al}_{0.97} \mathrm{Sn}_{0.03}\right)$, the Special Quasirandom Structure (SQS) [35] method was implemented to build the supercell. Formation energies are calculated using the formula exposed in Ref. [31] taking into account the corrections related to charged defects as detailed in [36]. On the one hand, Table 1 unambiguously shows that Ta substitutes $\mathrm{V}$, in agreement with the literature [4]. Its low formation energy indicates that the substituted compound could be thermodynamically stable at finite temperature and its zero charge points towards its doping inefficiency. On the other hand, the formation energy of Sn is larger than Ta but its solid solution behaviour for $x_{d e f}=0.03$ is well established by our experiments, which also confirm its electron donor character when substituting $\mathrm{Al}$.

The calculated and measured lattice thermal conductivity of $\mathrm{Fe}_{2} \mathrm{~V}_{0.94} \mathrm{Ta}_{0.06} \mathrm{Al}$ and $\mathrm{Fe}_{2} \mathrm{~V}_{0.97} \mathrm{Ta}_{0.03} \mathrm{Al}_{0.97} \mathrm{Sn}_{\mathrm{S}}$ are compared to each other in Figure 5 (left panel), whereas they are compared to the values calculated for perfect $\mathrm{Fe}_{2} \mathrm{VAl}$ in the inset. As expected, the substituted compounds exhibit a strong reduction from $\sim 60 \mathrm{~W} \mathrm{~m}^{-1} \mathrm{~K}^{-1}$ at $300 \mathrm{~K}$ to $\sim 10 \mathrm{Wm}^{-1} \mathrm{~K}^{-1}$ of their lattice thermal conductivity relatively to perfect $\mathrm{Fe}_{2} \mathrm{VAl}$. It can also be noticed in Fig. 5 that a fairly 

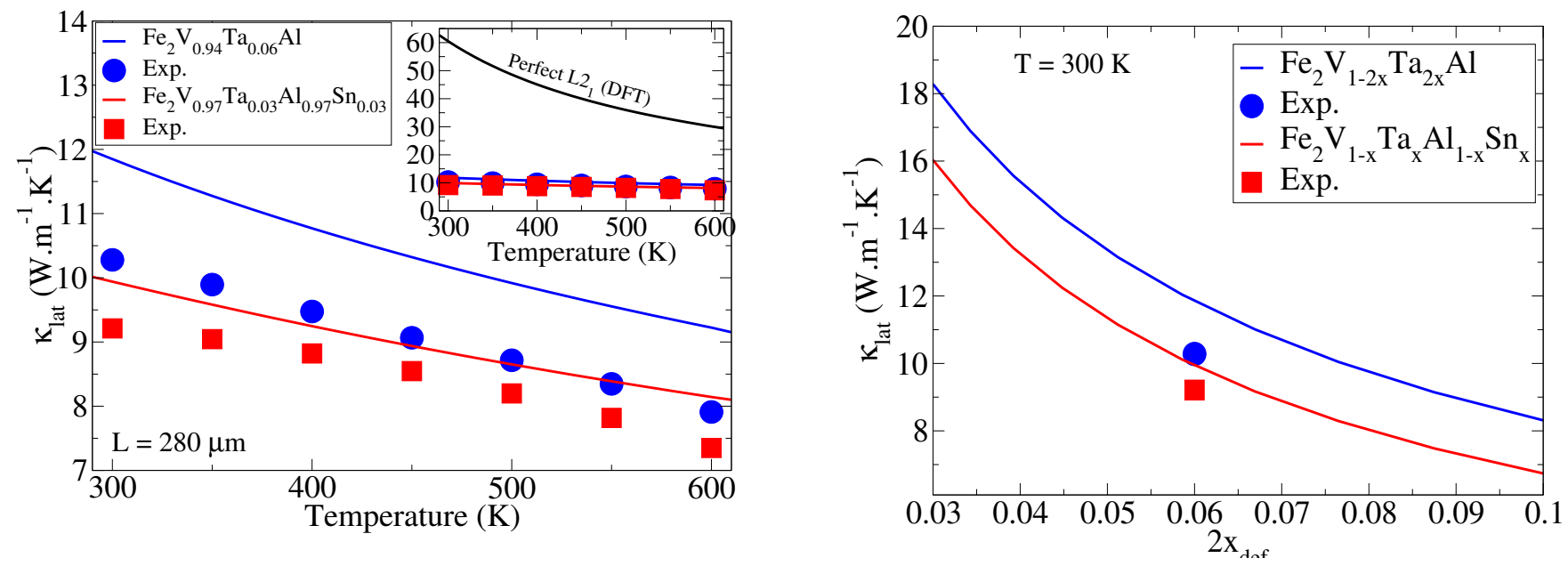

Figure 5: Evolution of the lattice thermal conductivity as a function of temperature (left panel) and total concentration of defects at $300 \mathrm{~K}$ (right panel) for substituted $\mathrm{Fe}_{2} \mathrm{VAl}$.

good agreement between calculations and experiments is obtained. It is worth noting that the calculated values are larger than the measured ones in both cases (more so in the Ta only subsituted sample). This most likely arises from the $\mathrm{Al} / \mathrm{V}$ inversions which have been neglected in the calculations and are nonetheless probably present in the experimental samples. For both the calculated and measured data, the lattice thermal conductivity is smaller in $\mathrm{Fe}_{2} \mathrm{~V}_{0.97} \mathrm{Ta}_{0.03} \mathrm{Al}_{0.97} \mathrm{Sn}_{0.03}$ than in $\mathrm{Fe}_{2} \mathrm{~V}_{0.94} \mathrm{Ta}_{0.06} \mathrm{Al}$. This is consistent with the fact that the relative mass difference which contributes to the perturbation potential $\left(\hat{V}_{M}\right)$ in equation $(1)$, is larger for $\mathrm{Al}$ and $\mathrm{Sn}(\Delta \mathrm{M} / \mathrm{M}=3.40)$ than for $\mathrm{V}$ and $\mathrm{Ta}(\Delta \mathrm{M} / \mathrm{M}=2.55)$. The changes in the values of the interatomic force constants, which are introduced by the substitution(s) and contribute to the perturbation potential $\left(\hat{V}_{K}\right)$, do not modify the trend imposed by $\hat{V}_{M}$, the mass term of the perturbation potential. To fully investigate this decrease of $\kappa_{\text {lat }}$ caused by the $\mathrm{Ta}$ and $\mathrm{Ta}+\mathrm{Sn}$ substitutions, the calculated lattice thermal conductivity at $300 \mathrm{~K}$ is plotted for both cases in Fig. 5 (right panel), as a function of the total substituent concentration $\left(2 x_{d e f}\right)$. It ranges from $3 \%$ to $10 \%$, the reported solubility limit for Ta in $\mathrm{Fe}_{2} \mathrm{VAl}$ [4]. These calculations show that whatever the total concentration, the dual $\mathrm{Ta}+\mathrm{Sn}$ substitution more efficiently depresses the lattice thermal conductivity in $\mathrm{Fe}_{2} \mathrm{VAl}$ than the single Ta substitution. When compared to $\kappa_{\text {lat }}=28 \mathrm{Wm}^{-1} \mathrm{~K}^{-1}$ experimentally found in $\mathrm{Fe}_{2} \mathrm{VAl}$, a reduction of about $70 \%$ of $\kappa_{\text {lat }}$ can be reached for a total concentration of $\mathrm{Ta}+\mathrm{Sn}$ equal to $\sim 8 \%$. Ta was considered up to now as the best substituent to decrease the lattice thermal conductivity in $\mathrm{Fe}_{2} \mathrm{VAl}$ [37]. These results could hence open a new route for improving the thermoelectric figure of merit in $\mathrm{Fe}_{2} \mathrm{VAl}$ by substituting $\mathrm{Ta}+\mathrm{Sn}$.

\section{Conclusion}

Our ab initio calculations show that phonon scattering processes such as anharmonic phononphonon, isotope and boundary scattering are not sufficient to explain the measured lattice thermal conductivity in stoichiometric $\mathrm{Fe}_{2} \mathrm{VAl}$. $\mathrm{Al} / \mathrm{V}$ inversions, adding an extra (mass + IFC) fluctuation scattering mechanism, need to be considered in the calculations. These $\mathrm{Al} / \mathrm{V}$ inversions undeniably play a central role in reconciling the calculated and experimental lattice thermal conductivity of pristine $\mathrm{Fe}_{2} \mathrm{VAl}\left(28 \mathrm{~W} \mathrm{~m}^{-1} \mathrm{~K}^{-1}\right.$ at $\left.300 \mathrm{~K}\right)$. A fair agreement is also obtained between the concentration of $\mathrm{Al} / \mathrm{V}$ inversions theoretically required to reach the experimental value of $\kappa_{\text {lat }}(5 \%)$ and the concentration of $\mathrm{Al} / \mathrm{V}$ derived from Hall effect measurements $(1.9 \%)$. Our calculations of the lattice thermal conductivity in Ta- and (Ta $+\mathrm{Sn})$ substituted $\mathrm{Fe}_{2} \mathrm{VAl}$ are in fairly good agreement with experiments. Both calculations and experiments show that $\mathrm{Fe}_{2} \mathrm{~V}_{1-x} \mathrm{Ta}_{x} \mathrm{Al}_{1-x} \mathrm{Sn}_{x}(0.03 \leq 2 \mathrm{x} \leq 0.10)$ systematically displays a smaller 
$\kappa_{\text {lat }}$ than $\mathrm{Fe}_{2} \mathrm{~V}_{1-2 x} \mathrm{Ta}_{2 x} \mathrm{Al}$.

\section{Acknowledgements}

The authors acknowledge the funding of this work by the "Agence Nationale pour la Recherche" through the contract "LoCoThermH" (ANR-18-CE05-0013-01). M.T.N and P.J. thank Pr. Jesús Carrete for the use of the "defect-version" of the AlmaBTE code as well as for helpful discussions.

\section{References}

[1] Lon E. Bell, Cooling, Heating, Generating Power, and Recovering Waste Heat with Thermoelectric Systems, American Association for the Advancement of Science, 321, $58951457-$ 1461 (2008).

[2] Salvador, J. R., Thermoelectric Energy Conversion, edited by Funahashi, R., Woodhead Publishing, 609-632 (2021)

[3] Nishino, Y. and Tamada, Y., Doping effects on thermoelectric properties of the offstoichiometric Heusler compounds $\mathrm{Fe}_{2-x} V_{1+x} A l$, Journal of Applied Physics 115, 123707 (2014)

[4] Renard, Krystel and Mori, Arinori and Yamada, Yuichiro and Tanaka, Suguru and Miyazaki, Hidetoshi and Nishino, Yoichi, Thermoelectric properties of the Heusler-type $\mathrm{Fe}_{2} \mathrm{VTa}_{x} A l_{1-x}$ alloys, Journal of Applied Physics 115, 033707, (2014).

[5] Garmroudi, F. and Riss, M. and Parzer, M. and Reumann, N. and Müller, H. and Bauer, E. and Khmelevskyi, S. and Podloucky, R. and Mori, T. and Tobita, K. and Katsura, Y. and Kimura, K., Boosting the thermoelectric performance of $\mathrm{Fe}_{2} \mathrm{VAl}$-type Heusler compounds by band engineering, Physical Review B 103, 085202 - (2021)

[6] Goldsmid, HJ., The thermal conductivity of bismuth telluride, Proceedings of the Physical Society. Section B, 69, 203, (1956).

[7] Mikami, M. and Kinemuchi, Y. and Ozaki, K. and Terazawa, Y. and Takeuchi, T., Thermoelectric properties of tungsten-substituted Heusler $\mathrm{Fe}_{2} \mathrm{VAl}$ alloy, Journal of Applied Physics, 111, 093710, (2012).

[8] Nishino, Yoichi and Deguchi, S. and Mizutani, U. Thermal and transport properties of the Heusler-type $\mathrm{Fe}_{2} \mathrm{VAl}_{1-x} \mathrm{Ge}_{x}(0 \leq x \leq 0.20)$ alloys: Effect of doping on lattice thermal conductivity, electrical resistivity, and Seebeck coefficient, Physical Review B, 74, 115115 (2006).

[9] Masuda, S. and Tsuchiya, K. and Qiang, J. and Miyazaki, H. and Nishino, Y., Effect of high-pressure torsion on the microstructure and thermoelectric properties of $\mathrm{Fe}_{2}$ VAl-based compounds, Journal of Applied Physics, 124, 035106 (2018).

[10] Lu, Wenjia and Zhang, Wenqing and Chen, Lidong, Thermoelectric properties of $\left(\mathrm{Fe}_{1-x} \mathrm{Co}_{x}\right)_{2} \mathrm{VAl}$ Heusler-type compounds, Journal of Alloys and Compounds, 484, 812-815 (2009).

[11] Bandaru, Subrahmanyam and Katre, Ankita and Carrete, Jesús and Mingo, Natalio and Jund, Philippe, Influence of antisite defects on the thermoelectric properties of $\mathrm{Fe}_{2} \mathrm{VAl}$, Nanoscale and Microscale Thermophysical Engineering 21, 237-246 (2017). 
[12] Knapp, I. and Budinska, B. and Milosavljevic, D. and Heinrich, P. and Khmelevskyi, S. and Moser, R. and Podloucky, R. and Prenninger, P. and E. Bauer, Impurity band effects on transport and thermoelectric properties of $\mathrm{Fe}_{2-x} N i_{x} V A l$, Physical Review B, 96, 045204 (2017)

[13] Broido, David A. and Malorny, Michael and Birner, Gerd and Mingo, Natalio and Stewart, D.A., Intrinsic lattice thermal conductivity of semiconductors from first principles, Applied Physics Letters,91, 231922 (2007).

[14] Ward, Alistair and Broido, DA and Stewart, Derek A and Deinzer, Gernot, Ab initio theory of the lattice thermal conductivity in diamond, Physical Review B, 80,125203, (2009).

[15] Li, Wu and Mingo, Natalio, Thermal conductivity of bulk and nanowire InAs, AlN, and BeO polymorphs from first principles, Journal of Applied Physics,114, 183505, (2013).

[16] Berche, Alexandre and Noutack, Martin Talla and Doublet, Marie-Liesse and Jund, Philippe, Unexpected band gap increase in the $\mathrm{Fe}_{2}$ VAl Heusler compound, Materials Today Physics, 100203 (2020).

[17] Maier, S. and Denis, S. and Adam, S. and Crivello, J-C. and Joubert, J-M. and Alleno, E., Order-disorder transitions in the $\mathrm{Fe}_{2} \mathrm{VAl}$ Heusler alloy,Acta Materialia, 121, 126-136, (2016).

[18] Katcho, N.A. and Carrete, J. and Li, Wu and Mingo, N.,Effect of nitrogen and vacancy defects on the thermal conductivity of diamond: An ab initio Green's function approach, Physical Review B,90, 094117 (2014).

[19] Katre, Ankita and Carrete, Jesús and Mingo, Natalio, Unraveling the dominant phonon scattering mechanism in the thermoelectric compound ZrNiSn, Journal of Materials Chemistry A,4, 15940-15944, (2016).

[20] Lambin, Ph. and Vigneron, Jean-Pol.,Computation of crystal Green's functions in the complex-energy plane with the use of the analytical tetrahedron method, Physical Review B, 29, 3430, (1984).

[21] Mingo, Natalio and Esfarjani, Keivan and Broido, DA and Stewart, DA, Cluster scattering effects on phonon conduction in graphene, Physical Review B 81, 045408, (2010).

[22] http://www.almabte.eu/.

[23] De Laeter, John R and Böhlke, John Karl and De Bièvre, P and Hidaka, H and Peiser, HS and Rosman, KJR and Taylor, PD. Atomic weights of the elements. Review 2000 (IUPAC Technical Report), Pure and applied chemistry, 75, 683-800, (2003).

[24] Tamura, Shin-ichiro,Isotope scattering of dispersive phonons in Ge, Physical Review B 27, $858,(1983)$.

[25] Togo, Atsushi and Chaput, Laurent and Tanaka, Isao Distributions of phonon lifetimes in Brillouin zones, Phys. Rev. B, 91094306 (2015).

[26] Kresse, G. and Joubert, D., Self-interaction correction to density-functional approximations for many-electron systems, Phys. Rev. B: Condens. Matter Mater. Phys, 59, 17581775, (1999).

[27] Perdew, John P and Burke, Kieron and Ernzerhof, Matthias, Generalized gradient approximation made simple,Physical review letters, 77,3865, (1996) 
[28] Monkhorst, Hendrik J. and Pack, James D.,Special points for Brillouin-zone integrations, Physical Review B, 13, 5188, (1976).

[29] Togo, A. and Tanaka, I., First principles phonon calculations in materials science, Scr. Mater., 108, 1-5, (2015).

[30] Rouleau, O. and Alleno, E., Measurement system of the Seebeck coefficient or of the electrical resistivity at high temperature, Review of Scientific Instruments, 84 (10), 105103 (2013).

[31] Subrahmanyam Bandaru and Philippe Jund, Electronic structure of the Heusler compound $\mathrm{Fe}_{2} \mathrm{VAl}$ and its point defects by ab initio calculations, Physica Status Solidi B, 254, 1600441 (2017).

[32] Hurd, C. M., The Hall effect in metals and alloys, Plenum Press, New-York, (1972).

[33] Stern, Robin and Wang, Tao and Carrete, Jesús and Mingo, Natalio and Madsen, Georg $\mathrm{KH}$,Influence of point defects on the thermal conductivity in FeSi, Physical Review B, 97, 195201 (2018).

[34] Liao, Bolin and Lee, Sangyeop and Esfarjani, Keivan and Chen, Gang, First-principles study of thermal transport in $\mathrm{FeSb}_{2}$, Physical Review B, 89, 035108, (2014).

[35] Zunger, Alex and Wei, S-H and Ferreira, LG and Bernard, James E., Special quasirandom structures,Physical Review Letters, 65, 353 (1990).

[36] Berche, Alexandre and Jund, Philippe Thermoelectric power factor of pure and doped ZnSb via DFT based defect calculations, Phys. Chem. Chem. Phys. 2123056 (2019).

[37] Alleno, Eric, Review of the thermoelectric properties in nanostructured $\mathrm{Fe}_{2} \mathrm{VAl}$, Metals, 8, $864,(2018)$.

[38] Kimura, K and Yamamoto, K and Hayashi, K and Tsutsui, S and Happo, N and Yamazoe, S and Miyazaki, H and Nakagami, S and Stellhorn, JR and Hosokawa, S and others, Local structure and atomic dynamics in $\mathrm{Fe}_{2} \mathrm{VAl}$ Heusler-type thermoelectric material: The effect of heavy element doping, Physical Review B, 101, 024302, (2020). 